misdiagnosed as nightmares, and generally treated as a complex partial seizure of frontal lobe origin. (see Progress in Pediatric Neurology II, PNB Publ, 1994, pp 193-4). Attacks of PDC, unlike PKC, were not benefited by anticonvulsants. PKC is sometimes referred to as PKD, and is often associated with infantile convulsions and epilepsy.

\title{
PAROXYSMAL KINESIGENIC DYSKINESIA AND EPILEPSY
}

Clinical and linkage data in 11 families with paroxysmal kynesigenic dyskinesia (PKD), with or without infantile convulsions (IC), were studied at the University of Utah, Salt Lake City, and multiple international centers. These families of diverse ethnic backgound had 95 affected individuals, the majority having chromosome 16 as the major locus, with heterogeneity. Kinesigenic dyskinesia (PKD) occurred in 42, infantile convulsions (IC) in 37, and a combined phenotype in 16. Infantile convulsions were usually afebrile and developed between 3 and 18 months; they remitted by age 3 years without treatment. Episodes of PKD began at 6 io 23 years (average 11 years), and were triggered by sudden movement, stress, and anxiety; they were controlled by anticonvulsant drugs, and were less frequent after age 25 years. EEGs were generally normal. The PKD/IC syndrome spans the phenotypic spectrum between epilepsy and movement disorder. (Swoboda KJ, Soong B W, McKenna C et al. Paroxysmal kinesigenic dyskinesia and infantile convulsions. Clinical and linkage studies. Neurology July (2 of 2) 2000;55:224-230). (Reprints: Dr Kathryn J Swoboda, University of Utah School of Medicine, Department of Neurology, Room 3R210, 50 North Medical Drive, Salt Lake City, UT 84132).

COMMENT. In an editorial, Berkovic SF classifies the Paroxysmal kinesigenic dyskinesia with infantile convulsions as a "channelopathy." (Paroxysmal movement disorders and epilepsy. Links across the channel. Neurology July 2000;55:169-170). Other paroxysmal disorders implicating channelopathies and manifested by seizures and dyskinesias include febrile seizures (sodium channel mutations), benign familial neonatal convulsions (potassium channel mutations), and episodic ataxia type 2 (calcium channel mutations). Different mutations are responsible for the diverse phenotypes. With PKD/IC syndrome, a single mutation may cause age-dependent symptoms: convulsions in infancy and dyskinesia in adolescence.

Risperidone and withdrawal dyskinesia is reported in a 13-year-old boy with ADHD, conduct disorder, and psychotic features, treated with lithium, valproic acid, and risperidone. (Lore C. I Am Acad Child Adolesc Psychiatry August 2000;39:941). Two weeks after the resperidone was discontinued, a withdrawal dyskinesia manifested with mouth movements, neck twisting, and intermittent upward gaze. Although withdrawal dyskinesia may resolve within 6 weeks, the author raises concerns about the use of resperidone in ADHD children with ODD and $\mathrm{CD}$, but without psychosis.

\section{INTERNATIONAL PERSPECTIVE OF TOURETTE SYNDROME}

The findings from a multisite, international database of 3500 individuals with Tourette syndrome (TS) from 22 countries are reported from the British Columbia Children's Hospital, Vancouver, Canada. Males outnumbered female patients, the average male:female ratio being 4.3:1. Mean age at onset of tics is 6.4 years, and mean age at diagnosis is 13 years. Comorbidities are reported in $88 \%$, and the most common is ADHD with TS (60\%). Other comorbid symptoms include OCD (27\%), CD/ODD (15\%), mood disorder (20\%), LD (23\%), and stuttering (8\%). 